\title{
EDITORIAL
}

\section{Industrial Revolutions, Society and Decisions for Tomorrow}

There were many pre-industrial civilizations spread around the globe, and the day-to-day life was aligned with social and cultural attributes of the communities. Pre-industrial civilizations, dated back to many centuries, were regionalbased, and the unity and the brain power of early humans were the key to success in many tasks. However, owing to natural tendency of the mankind, to look for comfort, compare and compete with others, and search for opportunities, a transition from pre-industrial activities to new manufacturing processes became a necessity. The initial transition took place during the latter half of the $18^{\text {th }}$ century, and rural, agrarian societies in Europe and America were transformed into industrialized ones. Mechanization of production was the main outcome of this transformation, which was possible through use of steam power. This was a substantial change, and is now marked as the first industrial revolution as many revolutions, having different modes and different technological inputs, later succeeded it. The first industrial revolution was reported to have initiated in Great Britain, the most powerful country back then, and it spread towards America and other countries. However, development of chemical industries was not established during the first industrial revolution; Though noise pollution and heat pollution did occur to some extent during this period, environmental pollution, in particular water pollution, did not take place to a significant extent, and the humans freely enjoyed the environment.

As a result of mechanization, handcraft and handlooms started to be produced in mass quantities by machines in factories, and methodologies of metal extraction from their ores were developed. The greatest breakthrough of this revolution was the increased human productivity, which also led to many changes in the society. Many years later, developments, such as the steampowered ship and the locomotive led to further changes in the society because humans and goods could be moved to great distances in shorter periods of time, leading to the initiation of globalization.

The second industrial revolution was geared for rapid industrialization. It is thus known as the Technological Revolution, which began in the late $19^{\text {th }}$ century and continued into the early $20^{\text {th }}$ century. A tremendous increase in economic growth was recorded during the second industrial revolution. Living standards of communities improved significantly, especially in the newly industrialized countries, together with massive production of goods as a result of enhancement in productivity. Further, significant improvements in public health and sanitation that resulted from expansion of scientific medical knowledge reduced the extent of infection and fatality rates from many diseases during this time period.
During the second revolution, applications of basic sciences opened-up many avenues. Understanding and application of the principles of thermodynamics in industrial processes, and the application of chemistry for synthetic approaches; manufacturing of inorganic chemicals and organic dye industries, in particular aniline-based ones, were among the notable advances. More importantly, optimization of experimental and process parameters was initiated during this period. Such efforts led to optimization of composition of stainless steel and its mass production. Other technological advances recorded during the second revolution are the application of chemical principles for purification of valuable metals, such as chromium, molybdenum, titanium and vanadium. Such purified metals were then used for the production of alloys with specific characteristics targeting desired uses. Various Ni-Fe alloys, with corrosion resistant properties were also developed during the latter part of the $19^{\text {th }}$ century. Further, research on Ni-Mo alloys of different compositions became successful in the early $20^{\text {th }}$ century, which later led to the invention of Hastelloy, which is still being manufactured as a supper alloy with much improved corrosion resistant properties. This alloy can be used for the production of containers to store highly corrosive and reactive solutions. However, fine tuning of industrial processes through automation, and improvement of accuracy of experimental results were yet to be developed. Together with the developments of industrialization and urbanization during this revolution, uncontrolled disposal of industrial and household waste led to severe undesirable effects on human health. There were reports on the pollution of many rivers in industrialized countries beyond limits. This prompted many countries to develop additional rules and regulations to safeguard human health, and laws devoted to protect the natural landscapes and wildlife.

The third industrial revolution began in the latter part of the $20^{\text {th }}$ century with a breakthrough on automation using electronics and information technology. Consequently, automated production processes were possible without an input of human involvement in some instances. Introduction of robots to carry out programmed sequences further eradicated human intervention as a result of this revolution. Robotics has the potential to improve efficiency and enhance safety levels, and with the introduction of robotics technology, the life style and work practices of humans were further changed.

Green revolution which dominated the world during the latter period of the second industrial revolution and the initial period of the third introduced large-scale agriculture with extensive use of chemical pesticides and fertilizers, vast irrigation schemes and heavy machinery. Furthermore, 
high yielding cereal grains and hybrid crops together with the above developments paved the way towards an increased productivity of agricultural crops. However, detrimental environmental impacts of green revolution, such as pollution due to agrochemicals, loss of untargeted species, and loss of habitat for wildlife and biodiversity were unavoidable. Soil erosion, landslides and other unexpected natural disasters were the other adverse effects of green revolution. Prevalence of cancer and birth defects experienced by humans today are probably connected with agrochemicals introduced during the green revolution and later.

The fourth industrial revolution, which is named the digital revolution, is continuously taking place at present. It was in fact built on the $3^{\text {rd }}$ revolution, and started in the middle of the last century. Artificial intelligence was the key development in this revolution. Although we may not have thought of it, artificial intelligence is already around us; for example, remote monitoring of appliances and virtual mode of various activities. Progress in artificial intelligence has become substantial in recent years, as a result of increased computing power. Software programmes are now available for many scientific developments such as discovery of new drugs and business functions. Importantly, investigation of symbiosis among various components of the ecosystem, including microorganisms, human body, food stuff and buildings inhabited by us, has been possible.

The fourth industrial revolution led by digitalization, in a way, embraced the humanity more than the first three revolutions did. There are so many benefits and advantages of this revolution providing efficient services and has brought pleasure to the community. Undoubtedly, a large percentage of the global population is connected by mobile devices, with remarkable processing power and storage capacity. This revolution has the potential to raise global income levels and improve the quality of life of humans, if the technology is used properly. It is a known fact that many of us are not able to function anymore without digital technology. Those who have gained the most from it have been those who access and those who enjoy the digital world - probably the younger generation more than the older generation! It is a fact that digitalization has been the key to the success during the pandemic situation we are still experiencing; many professional, scientific and industrial activities would have otherwise been at a standstill if the fourth revolution had not been taken place.

There is a dark side to the fourth industrial revolution. Digitalization-oriented lifestyle could weaken human capabilities, such as the analyzing and thinking power. Strong bond the mankind has developed overtime with smartphones is a major drawback in this aspect. Unlimited addiction to it would change valuable qualities of humans, such as patience, kindness and sensitivity, ability of collaboration and teamwork, degree of planning, and more importantly, interpersonal relationships and interaction with the environment. Neither technological developments nor consequences resulted from technology are spontaneous. It is the mankind that is responsible for orchestrating all these revolutions, and that made them happen. Time is ripe for us to rethink and readjust on unlimited developments that may bring in undesirable consequences tomorrow from today's actions through the fourth industrial revolution. If not, the mankind will become a robot of technology. Instead, technology may be developed further to upgrade the quality of human lives. Should we wait to find out what will happen tomorrow? Or make the correct decisions today? The decision is up for us! The scientists.

Namal Priyantha Faculty of Science University of Peradeniya namalpriyantha@sci.pdn.ac.lk 